\title{
Die Kunst, den Tod ins Leben zu lassen
}

\section{Nathalie Zeindler}

Freie Journalistin

\author{
Das Sterben wird heutzutage weniger verdrängt, sondern öfter diskutiert. Zahlrei- \\ che Debatten drehen sich um die Sterbehilfe und um die Frage, was einen guten \\ Tod ausmacht. Zu diesem Thema fand Ende September eine Podiumsdiskussion in \\ der Zürcher Paulus-Akademie in Kooperation mit Alte Anatomie - Forum für Medi- \\ zin und Gesellschaft - statt.
}

«Denn wir sind nur die Schale und das Blatt. Der grosse Tod, den jeder in sich hat, das ist die Frucht, um die sich alles dreht», schrieb einst Rainer Maria Rilke, der sich immer wieder mit der Todesthematik und dem Identitätszerfall in seinen Werken befasst hat. Die literarische Beschäftigung mit dem Tod ist eine von vielen Ausdrucksformen. Wenn der Tod allerdings in greifbare Nähe rückt - ob unser eigener oder jener einer geliebten Person -, reagieren trotz allmählicher Enttabuisierung nicht wenige mit Ängsten und Hilflosigkeit. «Das lässt sich nur ändern, wenn wir den Tod nicht in den fiktionalen Raum abschieben", sagt Dr. Nina Streeck, Fachverantwortliche Ethik und Lebensfragen, Institut Neumünster ZH und Autorin des neu erschienenen Buches «Jedem seinen eigenen Tod».

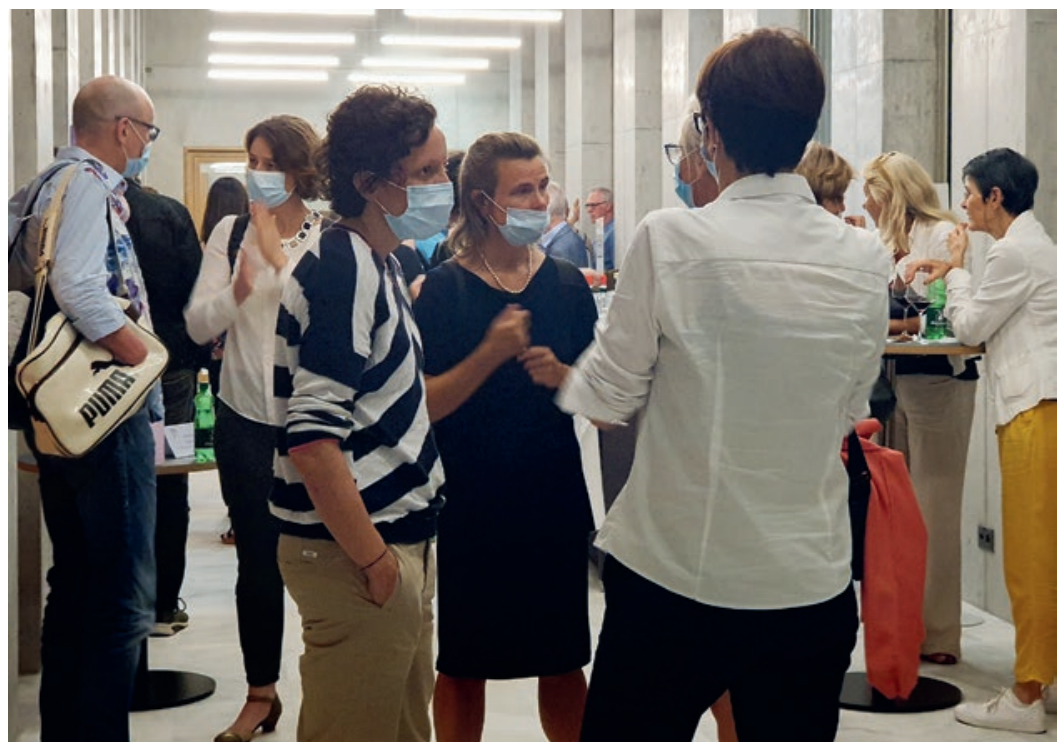

Angeregte Gespräche auch nach der Podiumsdiskussion in der Zürcher Paulus-Akademie.
Zur Verwirklichung eines sogenannten authentischen Sterbens stehen viele Möglichkeiten zur Verfügung. Manche treten einer Sterbehilfeorganisation bei, andere verfassen frühzeitig eine Patientenverfügung, um sicherzustellen, dass alles nach ihren Wünschen verläuft, sollten sie ihre Urteilsfähigkeit einmal verlieren.

\section{Unvorhergesehene Entwicklungen berücksichtigen}

«Es ist wichtig, die Bevölkerung darauf hinzuweisen, dass im Leben jederzeit etwas passieren kann und in einer urteilsfähigen Situation ein entsprechender Beschluss notwendig ist», sagt Dr. Peter Steiger, Stv. Direktor für Intensivmedizin UniversitätsSpital Zürich, im Rahmen der Podiumsdiskussion zum Thema "Jedem seinen eigenen Tod». Immer wieder erlebt er, dass klare Bestimmungen seitens der Patientinnen und Patienten fehlen und diese mit viel Aufwand und Ressourcen therapiert werden, obwohl sich hinterher herausstellt, dass die Betroffenen im Grunde ein anderes Vorgehen wünschten. Manchmal können aber auch andere Probleme zutage treten, nämlich dann, wenn der letzte Wille aus einer Laune heraus formuliert wurde und schliesslich von einer falschen Voraussetzung ausgegangen wird. «Es kann hilfreich sein, in digitalen Patientenverfügungen darzustellen, dass eine bestimmte Verfügung kenntnisreich unterschrieben wurde. Dadurch können auch jene Personen unterstützt werden, welche eine schwierige Entscheidung treffen müssen", so Prof. Dr. Nikola Biller-Andorno, Direktorin des Instituts für Biomedizinische Ethik und Medizingeschichte UZH. 


\section{Der Tod hat viele Gesichter}

Wie sieht es mit jenen Personen aus, die den Entwicklungen lieber ihren Lauf lassen möchten, ohne einzugreifen? Podiumsteilnehmer Dr. Roland Kunz, Chefarzt Universitäre Klinik für Akutgeriatrie und ärztlicher Leiter Zentrum für Palliative Care Stadtspital Waid und Triemli ZH, fügt an: «Palliative Care soll nicht als Sterbeideal gelten. Vielmehr muss die Möglichkeit eröffnet werden, dass jeder so sterben kann, wie er es für richtig hält. Wichtig ist, den Kranken dort abzuholen, wo er steht, und ihn ein Stück weit auf diesem Weg zu begleiten.» Es komme auch immer wieder vor, dass Patienten ihr Lebensende bis zum letzten Atemzug nicht akzeptieren können, was auch eine legitime Haltung sei. Wenn eine Terminalpatientin beispielsweise zwei schulpflichtige Kinder habe, dürfte es wohl mehr als nachvollziehbar sein, wenn sich diese mit dem Tod nicht versöhnen könne.

Die Palliativmedizin steht mit Wunscherfüllung in direktem Zusammenhang - im Gegensatz zur Intensivmedizin, die meist nicht mehr ansprechbare Personen behandelt. «Wir müssen uns darauf verlassen, was uns die Angehörigen erzählen und was der Patient einst vermittelt hat. Auch handeln Angehörige oft aus der eigenen Betroffenheit heraus, was Konfliktpotenzial mit sich bringt und Gespräche mit Ethikmedizinern voraussetzt», erklärt Dr. Peter Steiger. Während sein Fachgebiet klare Regelungen als besonders wichtig erachtet, zielt die Palliativmedizin auf ein Sterben unter Miteinwirkung der Betroffenen in Form einer Verbesserung der Lebensqualität $a b$.

Im Umgang mit dem Sterben diente der Autorin Nina Streeck der Authentizitätsbegriff als Diagnosekonzept und damit auch eine bestimmte Vorstellung von einem guten Hinschied, der sich dadurch auszeichnet, dass der Mensch zum Ausdruck bringen kann, wer er ist und sein möchte.

\section{Individualität im Fokus}

Dr. Kunz erzählt von einer Patientin, einer krebskranken Frau, die sich schon vor Jahren vehement für ein selbstbestimmtes Ableben aussprach. Als sich ihr Zustand rasch verschlechterte und sie realisierte, dass der Tod unmittelbar vor der Tür stand, kamen Stressgefühle auf, weil sie eigentlich selbst entscheiden wollte, wann es Zeit sei zu gehen. «Unsere Aufgabe bestand darin, ausführlich mit ihr zu sprechen und ihr eine innere Gelassenheit näherzubringen, um das Sterben anzunehmen, was auch gelang.» Wer den Tod zulässt, verliert nicht sein Gesicht, denn es handelt sich ebenfalls um eine Form der Selbstbestimmung. Im Falle einer Demenz zeichnet sich bereits langfristig

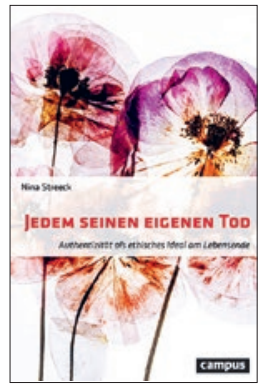

\author{
Nina Streeck \\ Authentizität als ethisches \\ Ideal am Lebensende \\ Frankfurt am Main: \\ Campus Verlag; 2020 \\ 357 Seiten \\ ISBN 978-3-593-51235-8
}

Jedem seinen eigenen Tod

$a b$, dass eine Person dereinst nicht mehr über ein kohärentes Verständnis ihrer selbst verfügt. Podiumsteilnehmer Michael Schmieder, Demenzexperte und Verwaltungsrat Stiftung Sonnweid: «Der Tod ist dann gut, wenn die Betroffenen auf irgendeine Art dazu bereit sind, wenn auch nicht bewusst. In einem fortgeschrittenen Demenzstadium ist zudem nicht mehr klar, ob der Patient noch weiss, dass er lebt. Es handelt sich um eine Phase zwischen Leben und Tod.»

Die grösste Herausforderung im authentischen Umgang mit dem eigenen Sterben bestehe darin, «zum Ausdruck zu bringen, wie man sich mit Blick auf den herannahenden Selbstverlust versteht», so Nina Streeck.

\section{Carpe Diem}

Welche Haltung jemand in Bezug auf schwindende Fähigkeiten einnimmt, spielt keine Rolle. Vielmehr ist von Bedeutung, ob der oder die Betroffene bis zum letzten Atemzug mit sich selbst im Einklang bleiben kann. «Eine solche Denkweise könnte leichter umzusetzen sein, wenn man jeden Tag bewusst lebt, solange dies möglich ist, und sich irgendwann damit abfindet, dass das Leben aufhört», meint Dr. Steiger mit Nachdruck. Und hier erlangt Nina Streecks Authentizitätsideal durchaus einen Sinn. Zwei Beispiele, die Vorsorgemassnahmen und der begleitete Suizid kommen als glaubwürdiges Sterben in Betracht, weil sie eine Haltung des Gestaltens und des Planens bedeuten. Ein selbstbestimmter Tod verwirklicht sich aber nicht nur dann, wenn eine Person bis zuletzt entscheiden kann, sondern auch, wenn sie unter anderem auch den körperlichen und geistigen Abbau geschehen lässt. Problematisch dürfte es hingegen sein, wenn ein verstecktes Urteil transportiert wird, welche Sterbeweise als «ideal» gilt. Die Antwort nach dem guten Sterben bleibt deshalb mit offenen Fragen verbunden. Seine wahre Stärke entfaltet besagtes Ideal als kritischer Begriff, denn der Eroberung neuer Freiheiten folgen neue Zwänge, die es seinerseits zu überbieten gilt. Nina Streeck: «Die Bitte um den 'eigenen' Tod verstummt nicht. Vielmehr müssen die eigenen Idealvorstellungen immer wieder reflektiert werden.» 